\title{
Mortalidade por asma no Município de São Paulo, 1993 a 1995: análise por causa múltipla de morte
}

\author{
Asthma mortality in the Municipality \\ of São Paulo (1993-1995): \\ analysis by multiple cause of death
}

Evani Marzagão Beringhs Rio ${ }^{1}$

Paulo Rogério Gallo 1

Alberto Olavo A. Reis 1

1 Departamento de Saúde Materno-Infantil, Faculdade de Saúde Pública, Universidade de São Paulo. Av. Dr. Arnaldo 715, São Paulo, SP 01246-904, Brasil. evani@beringhs.com.br prgallo@usp.br
Abstract This article aims to quantify the asthma mortality rate using the multiple causality analytical method in comparison to the underlying cause of death. Data were obtained from all death certificates $(224,854)$ of individuals from 5 to 34 years of age issued in 1993, 1994, and 1995 in the Municipality of São Paulo, in which mention of the word asthma was considered valid information (code 493/ICD-9). First, the underlying cause of death was studied. The same data were then used, but submitted to the multiple causality method. The comparative study of basic and multiple causes of death increased the diagnosis of asthma by a ratio of 1.165. The over-20-year age group remained the most affected by the problem. The age range of individuals most affected by this disease did not change with the analytical method (20 to 34 years). Although asthma was present more frequently when the multiple causality method was used, the increase in the number of asthma diagnoses highlights that the disease can be considered underreported in Brazil in the chain of events leading to death.

Key words Asthma; Mortality; Risk Factors; Cause of Death

Resumo Este trabalho teve como objetivo quantificar as taxas de mortalidade por asma, utilizando o método de análise por causa múltipla de morte e comparando com aquelas obtidas pelo método da causa básica. Para isso, selecionou-se todos os Atestados de Óbito (224.854), nas idades entre 5 a 34 anos, que continham em qualquer das partes a citação de asma, código 493 (CID-9), nos anos de 1993/1994/1995, no Município de São Paulo e aplicou-se o método de Tabulação de Causas Múltiplas de Morte (TCM). Comparou-se então as taxas de notificação da asma obtidas nos dois métodos de análise: como causa básica e como causa associada. O estudo comparativo mostrou que o método de TCM elevou o diagnóstico de asma na razão de 1 para 1,165. A faixa etária acima dos vinte anos foi a mais atingida nas duas formas de análise, ou seja, a faixa etária não se alterou pelo método de tabulação e foi mais alta dos 20 aos 34 anos. O aumento encontrado no número de diagnósticos, mostrou que a asma, embora mais presente ao associar-se à metodologia da multicausalidade do óbito, ainda pode ser considerada como pouco notificada na seqüência de eventos que levam o indivíduo à morte.

Palavras-chave Asma; Mortalidade; Fatores de Risco; Causa da Morte 


\section{Introdução}

A asma é considerada como uma das mais importantes doenças respiratórias. De características crônicas, atinge aproximadamente 5\% da população americana (Weiss \& Wagener, 1990).

Burney (1988) e Hunt et al. (1993) relatam aumento na morbidade e mortalidade por asma em diversos países. Em nosso meio, apesar da alta incidência relatada nas estatísticas de atendimento ambulatorial e em alguns estudos de morbidade (Campos, 1997; Kamoi et al., 1998), esse aumento não parece refletir na mortalidade.

No Rio Grande do Sul, as taxas de mortalidade variaram no período de 1970 a 1992 entre 0,040 e 0,689/ 100 mil conforme o ano e as faixas etárias (Chatkin et al., 1997). Rio et al. (2002) estudaram a mortalidade por asma no Município de São Paulo, em dois triênios: 1983/1985 e 1993/1995, considerando apenas a causa básica de morte, e não constataram o aumento da mortalidade neste período. Neste estudo, o coeficiente de mortalidade específico por 100 mil habitantes foi de 0,630 para o primeiro período e de 0,608 para o segundo. Estes resultados vieram reafirmar os relatos de Lotufo et al. (1995) e Campos (1997), que comprovaram a estabilidade destas taxas.

Entretanto, alguns autores argumentam que a prática de preenchimento incompleto e incorretos das Declarações de Óbito (DO), poderia dificultar a análise dos dados nele contidos, podendo levar à discordância entre os resultados (Heckmann et al., 1989; Mendonça et al., 1994). Segundo Siqueira et al. (1999), o preenchimento incorreto não afetaria as taxas de mortalidade para o capítulo das doenças respiratórias da Classificação Internacional de Doenças (CID).

Por outro lado, em estudo clássico, Laurenti (1981) alertou para a possibilidade de subnotificação de doenças crônicas em detrimento das agudas como causa de morte. Sob este ponto de vista, a asma poderia estar sendo notificada como causa associada e não como causa básica de morte, razão pela qual considerou-se importante quantificá-la por meio do método de avaliação do conjunto de causas que contribuíram para o óbito (causas múltiplas), objetivo do presente trabalho. Além disso, pretende-se realçar a importância das informações geradas por dados secundários na formulação das políticas públicas.

\section{Metodologia}

Estudou-se o universo das DOs do triênio 1993/ 1995 ( $n=224.854$ ), comparando as distribuições de freqüência da notificação de asma como causa básica e causa associada, dentre as múltiplas causas que concorreram para os óbitos. Os coeficientes foram padronizados pelo método direto. A conveniência do período de análise foi definida após pré-avaliação da estabilidade e confiabilidade dos dados do sistema ACME (Automated Classification of Medical Entities), instituído pela Fundação Sistema Estadual de Análise de Dados (Fundação Seade) em 1983. O banco de dados foi transformado pelo método da Tabulação de Causas Múltiplas de Morte (TCM), possibilitando a avaliação de todas as causas contidas nas DOs (Santo \& Pinheiro, 1999). Foram considerados os dados referentes ao diagnóstico de asma como causa básica e/ou associada, pelo código 493, referente à CID em sua 9a revisão (OMS, 1979). A faixa etária escolhida para análise foi de 5 a 34 anos, inclusive. Os limites foram estabelecidos em função de dificuldades diagnósticas nas idades anteriores e posteriores (Hunt et al., 1993; Kamoi et al., 1998). Por se tratar do universo das DOs, a análise dos valores apresentados nas tabelas são feitos por comparação direta dos coeficientes e taxas.

\section{Resultados e discussão}

A Tabela 1 mostra que o diagnóstico de asma aparece mencionado 116 vezes em qualquer parte da DO, sendo 103 vezes citada como causa básica. Observa-se, portanto, a elevação à razão de 1:1,126 representando uma proporção de $88,79 \%$ que indiretamente, confirma a pouca notificação da asma como causa associada. Convém realçar que Laurenti (1981), mostrou que a cobertura de óbitos no Município de São Paulo é próxima a $100 \%$, contrastando com outras regiões do país. A diferença de 11,21 \% entre as classificações deve ser então valorizada, uma vez que se trata do universo dos óbitos do município. Além destes pontos, pode-se notar que a tendência do aumento da mortalidade por asma nos adultos de 20 a 34 anos, não foi alterada pelo método de análise das causas múltiplas.

Não observamos em nosso meio, mesmo considerando a maior proporção encontrada na análise de causas múltiplas de morte, uma taxa de mortalidade por asma que se assemelhe às taxas notificadas nos Estados Unidos, cerca de 2,7 vezes maiores para o mesmo pe- 
Distribuição dos óbitos e dos coeficientes de mortalidade específica (CME) por asma1, como causa básica (CB)

e causa múltipla (CM), segundo faixa etária, no triênio 1993/1995, no Município de São Paulo, Brasil.

\begin{tabular}{lccccc}
\hline $\begin{array}{l}\text { Faixa } \\
\text { etária }\end{array}$ & $\begin{array}{c}\text { Óbitos } \\
\text { (CB) }\end{array}$ & $\begin{array}{c}\text { Óbitos } \\
\text { (CM) }\end{array}$ & $\begin{array}{c}\text { População } \\
\text { estimada }\end{array}$ & $\begin{array}{c}\text { CME } \\
\text { (CB) }\end{array}$ & $\begin{array}{c}\text { CME } \\
(\text { CM) }\end{array}$ \\
\hline $5-9$ & 6 & 8 & 2.958 .625 & 0,236 & 0,270 \\
$10-14$ & 21 & 21 & 2.851 .160 & 0,736 & 0,736 \\
$15-19$ & 12 & 13 & 2.665 .154 & 0,450 & 0,488 \\
$20-24$ & 19 & 23 & 2.877 .533 & 0,623 & 0,799 \\
$25-29$ & 19 & 25 & 2.911 .458 & 0,721 & 0,858 \\
$30-34$ & 26 & 26 & 2.686 .858 & 0,893 & 0,968 \\
Total & 103 & 116 & 16.950 .788 & 0,608 & 0,684 \\
\hline
\end{tabular}

1 Óbitos por 100 mil habitantes.

2 Dados do IBGE.

Fonte: Fundação Seade.

ríodo (CDC, 2003) ou mesmo em relação a outros países (Burney, 1988; Weiss \& Wagener, 1990). Assim, é preciso supor que em nossa realidade, ou estamos atendendo e tratando devidamente todos os acometidos, ou a asma estaria ainda sendo subnotificada. Possibilidade esta reforçada pela alta freqüência encontrada na prática clínica, no número de internações e no atendimento em prontos-socorros.
É preciso ainda destacar que a asma necessita, seja no seu diagnóstico, no seu controle ou pelas suas conseqüências para a saúde do indivíduo, de uma constante atenção dos profissionais da área de saúde para reduzir o número de acometidos e de suas seqüelas, lembrando que os óbitos por asma são tidos como "evento sentinela" em Saúde Pública, ou seja, são mortes que poderiam ser evitadas.

\section{Referências}

BURNEY, P., 1988. Asthma deaths in England and Wales, 1931-85: Evidence for a true increase in asthma mortality. Journal of Epidemiology and Community Health, 42:316-320.

CAMPOS, H. S., 1997. O peso da asma. Boletim de Pneumologia Sanitária, 5:40-58.

CDC (Centers for Disease Control and Prevention), 2003. A Presentation on Asthma Management and Prevention. 25 March 2003 <http://www.cdc.gov/ nceh/airpollution/asthma/speakit/epidemiology. ppt>.

CHATKIN, J. M.; BARRETO, S. M.; FONSECA, N. A.; GUTIERREZ, C. A. \& SEARS, M. R., 1997. Trends in asthma mortality in young people in southern Brazil. Annals of Allergy, Asthma \& Immunology, 82:287-292.

HECHMANN, C. I.; CANARI, L. H.; SANT'ANA, U. L. \& BORDIN, R., 1989. Análise do preenchimento de declaração de óbitos em localidades do Estado do Rio Grande do Sul (Brasil). Revista de Saúde Pública, 23:292-297. 
HUNT, L. W.; SILOUNSTEIN, M. D.; RIED, C. E.; O'CONELL, E. J.; O'FALLON, W. M. \& YUGINGEER, J. W., 1993. Accuracy of the death certificate in a population-based study of asthmatic patients. JAMA, 269:1947-1952.

KAMOI, O. T.; ROSÁRIO, N. A. \& FARIAS, L., 1998. História natural da asma em crianças: Há remissão na adolescência? Revista de Pediatria, 20:310-313.

LAURENTI, R., 1981. Doenças respiratórias como causa de morte no Município de São Paulo, SP (Brasil). Revista de Saúde Pública, 15:353-363.

LOTUFO, P. A.; BEUSEÑOR, I. J. M. \& LOLIO, C. A., 1995. Mortality from asthma in the state of $S$. Paulo, Brazil (1970-1972). Revista de Saúde Pública, 29:434-439.

MENDONÇA, E. F; GOULART, E. M. A. \& MACHADO, J. A. D., 1994. Confiabilidade de declaração de causa básica de mortes infantis em região metropolitana do Sudeste do Brasil. Revista de Saúde Pública, 28:385-391.
OMS (Organização Mundial da Saúde), 1979. Manual de Classificação de Doenças, Lesões e Causas de Óbitos: 9a Revisão, 1975. São Paulo: Centro da OMS para Classificação de Doenças em Português.

RIO, E. M. B.; GALLO, P. R. \& SIQUEIRA, A. A. F., 2002. Mortalidade por asma no Município de São Paulo. Revista de Saúde Pública, 36:149-154.

SANTO, A. H. \& PINHEIRO, C. E., 1999. Tabulador de causas múltiplas de morte. Revista Brasileira de Epidemiologia, 2:1-2.

SIQUEIRA, A. A. F.; RIO, E. M. B.; TANAKA, A. C. D.; SCHOR, N.; ALVARENGA, A. T. \& ALMEIDA, L. C., 1999. Mortalidade feminina na região sul do Município de São Paulo: Qualidade da certificação médica dos óbitos. Revista de Saúde Pública, 33: 499-504.

WEISS, K. B. \& WAGENER, D. K., 1990. Changing patterns of asthma mortality. Identifying target populations at high risk. JAMA, 264:1683-1687.

Recebido em 13 de março de 2002

Versão final reapresentada em 24 de abril de 2003

Aprovado em 3 de junho de 2003 